Esta revista forma parte del acervo de la Biblioteca Jurídica Virtual del Instituto de Investigaciones Jurídicas de la UNAM

\title{
LOS CUERPOS AUXILIARES DE SEGURIDAD PÚBLICA DEL ESTADO DE MÉXICO Y LAS AFECTACIONES AL DERECHO DE ACCESO A LA INFORMACIÓN PÚBLICA Y A LA PROTECCIÓN DE DATOS PERSONALES
}

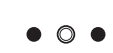

\author{
José Guadalupe LUNA HERNÁNDEZ*
}

\section{Planteamiento del problema}

En el Estado de México existe un fuerte debate provocado por la existencia de cuerpos auxiliares de seguridad pública que realizan funciones de guardia y custodia de instituciones y particulares a cambio de una contraprestación económica por sus servicios. La actividad que desarrollan es consecuencia de la autorización emitida por las autoridades del estado al reconocerlos como organismo auxiliar. ${ }^{1}$ Este problema es cada vez más visible como consecuencia de la expansión de sus actividades, durante la actual administración federal, una vez que elementos de uno de estos organismos sustituye a los elementos de la policía bancaria del Distrito Federal, quienes tradicionalmente custodiaban las instalaciones de las dependencias de la administración pública federal.

* Es comisionado en el Instituto de Transparencia, Acceso a la Información Pública y Protección de Datos Personales del Estado de México y Municipios (Infoem) desde julio de 2015; maestro en derecho constitucional y derechos humanos por la Universidad Panamericana; licenciado en ciencia política y administración pública por la Facultad de Ciencias Políticas y Sociales de la UNAM; jose.luna@itaipem.org.mx.

Fecha de recepción: 13 de diciembre de 2016.

Fecha de dictamen: 21 de marzo de 2017.

1 Compartiendo incluso la licencia para la portación de armas que la Sedena emitió al gobierno estatal (El Informador, 2011), sin que ninguna autoridad realice formalmente funciones de control o supervisión sobre los ingresos o el funcionamiento de dichos organismos, cuyos integrantes se han visto involucrados en hechos delictivos (Villamil, 2013), lo que desde luego ha provocado inquietudes entre los diputados locales de diversas legislaturas (Poder Legislativo del Estado de México, 2015: 143; Villamil, 2013) y en la opinión pública (Alfa Diario, 2016). 
Esta revista forma parte del acervo de la Biblioteca Jurídica Virtual del Instituto de Investigaciones Jurídicas de la UNAM

Los derechos de acceso a la información pública y protección de datos personales han sido ejercidos, por diversas personas, para acceder a información en posesión de dicho organismo, a través de la Secretaría de Seguridad Ciudadana, primero, y de la Comisión de Seguridad Ciudadana, después. Se requiere el acceso a información que presumen pública o a sus datos personales, generados y administrados por los organismos auxiliares. En todos estos casos, la mayoría del Pleno del órgano garante en la materia, el Instituto de Acceso a la Información Pública y Protección de Datos Personales del Estado de México y Municipios (Infoem), ha ratificado los argumentos del sujeto obligado que niegan el acceso a la información o a los datos personales, lo que, como se mostrará, afecta gravemente el régimen jurídico que tutela dichos derechos humanos.

\section{LOS RECURSOS RESUELTOS}

El 5 de agosto de 2015, el Pleno del Infoem resolvió los recursos 1129/ INFOEM/IP/RR/2015 y 1130/INFOEM/AD/RR/2015, confirmando la respuesta de la Secretaría de Seguridad Ciudadana que se negó a entregar documentos de alta y baja de una persona del Cuerpo de Vigilancia Auxiliar y Urbana del Estado, fundándose para ello en el artículo 103 de la Ley de Seguridad del Estado, 8o., fracción XV, de la Ley que crea la Comisión Estatal de Seguridad Ciudadana del Estado de México, y 8o., fracción XXIII, y 10, fracción XV, del Reglamento Interior de la propia Secretaría, en virtud de que la relación entre dicha dependencia y el organismo auxiliar es sólo "de carácter operativo y en situaciones de urgencia o desastre", ya que no depende "orgánica, presupuestal, programática ni administrativamente del sujeto obligado”. En esta ocasión, el Pleno consideró que los integrantes de los Cuerpos de Seguridad no son servidores públicos del gobierno del Estado de México y, en consecuencia, según el dicho del sujeto obligado, la información no obra en su poder.

El 03 de mayo de 2016 se resolvió el recurso de revisión 723/INFOEM/ IP/RR/2016, modificando la respuesta de la Comisión Estatal de Seguridad Ciudadana que se negó a entregar el número de guardias, el nombre del titular o responsable de los Cuerpos de Seguridad Auxiliares del Estado de México, catálogo general de bienes muebles, especificando vehículos automotores y placas, así como los servicios que ofrece y sus tarifas, utilizando el mismo argumento señalado en el párrafo anterior y fundándose en el artículo 103 de la Ley de Seguridad del Estado de México, 3o., fracción XV, de la Ley que crea la Comisión Estatal de Seguridad Ciudadana 
Esta revista forma parte del acervo de la Biblioteca Jurídica Virtual del Instituto de Investigaciones Jurídicas de la UNAM

del Estado de México, y 10, fracción XXII, del Reglamento Interior de dicha Comisión. En esa ocasión, el Pleno ordenó la entrega del número de guardias y el nombre del titular en virtud de que la relación que existe es operativa y en casos de desastre. La resolución fue aprobada por mayoría de votos con un voto disidente. Antes de esta resolución, la mayoría había rechazado un proyecto que ordenaba entregar toda la información.

El 15 de junio de 2016 se resolvió el recurso de revisión 1522/INFOEM/ IP/RR/2016, confirmando la respuesta de la Comisión Estatal de Seguridad Ciudadana que se negó a entregar el contrato de prestación de servicios firmado por el mismo organismo auxiliar y una asociación de colonos, así como programas, operativos, protocolos y directrices, utilizando el mismo argumento descrito en los párrafos anteriores y la fundamentación referida en el anterior. El Pleno razonó su decisión en la propia respuesta del sujeto obligado, consistente en que los cuerpos auxiliares de seguridad no cuentan con una dependencia orgánica, presupuestal, programática ni administrativa de la autoridad. La resolución fue aprobada por mayoría con un voto disidente.

El 31 de agosto de 2016 se resolvió el recurso de revisión 2014/INFOEM/IP/RR/2016, confirmando la respuesta de la Comisión Estatal de Seguridad Ciudadana que se negó a entregar el expediente personal de un exelemento, en posesión del Cusaem (se trata de una persona en prisión), para lo cual se utilizó el mismo argumento y la fundamentación que se han señalado en los párrafos anteriores. El Pleno fundamentó su razonamiento en virtud de que dichos cuerpos auxiliares de seguridad no cuentan con una dependencia orgánica, presupuestal, programática ni administrativa, como refirió el sujeto obligado. La resolución fue aprobada por mayoría con un voto disidente.

Por último, el 5 de octubre de 2016 se resolvieron los recursos de revisión 2756/INFOEM/IP/RR/2016 y 2757/INFOEM/IP/AD/2016, sobreseyendo el recurso porque en la fase de alegatos la Comisión Estatal de Seguridad Ciudadana abundó en información que precisa que no existe una dependencia orgánica, presupuestal, programática ni administrativa entre el sujeto obligado y el Cuerpo de Guardias de Seguridad Industrial, Bancaria y Comercial del Valle de Cuautitlán Texcoco, argumento que le bastó a la mayoría del Pleno para considerar que había cesado toda controversia y que, en consecuencia, era adecuada la negativa de la autoridad para entregar los talones de pago de una persona. La resolución fue aprobada por mayoría con un voto disidente.

$\mathrm{Al}$ emitir dichas resoluciones, el órgano garante aceptó que los sujetos obligados del Estado de México, bajo su jurisdicción, no cuentan con una 
Esta revista forma parte del acervo de la Biblioteca Jurídica Virtual del Instituto de Investigaciones Jurídicas de la UNAM

relación administrativa que les permita conocer algo más que el número de elementos que integran a estos organismos auxiliares y el nombre de su mando operativo.

\section{RAZONES POR LAS CUALES LAS RESOLUCIONES EMITIDAS AFECTAN EL RÉGIMEN JURÍDICO DE TUTELA DE DICHOS DERECHOS}

Las razones y circunstancias que explican el sentido de las resoluciones emitidas por la mayoría del Pleno del órgano garante del Estado de México - son ajenas al régimen jurídico que protege los derechos de acceso a la infor- mación pública y a la protección de datos personales, y se circunscriben a una tendencia gubernamental que ha prevalecido y que sólo puede responder al interés de ocultar ingresos que, en 2011, antes de que se encargaran de la custodia de las instalaciones que ocupan las dependencias federales, ascendían a dos mil millones de pesos, ${ }^{2}$ y cuya administración y destino se mantiene en un marco de total discrecionalidad, ya que ni el órgano de fiscalización del estado ha podido auditar dichos fondos. Y ahora que las personas pretenden acceder a información pública o a los datos personales que se encuentran en su posesión, se niega el acceso con la finalidad de mantener en su actual estado de opacidad la operación de dichas corporaciones.

De los antecedentes jurídicos y administrativos de este organismo auxiliar puede apreciarse que en el pasado hubo una clara relación de dependencia con las instituciones públicas, tan es así que el Reglamento de los Cuerpos de Seguridad Pública del Estado de México, emitido por el gobernador Alfredo Baranda y vigente desde el 11 de junio de 1987 y hasta por lo menos 1994 y 1995 (fecha en la cual se generaron los datos personales a los que pretendía acceder uno de los recurrentes), precisaba una relación directa y de dependencia clara e indubitable entre los Cuerpos Auxiliares y la, en ese entonces, Dirección General de Seguridad Pública y Tránsito del Estado de México, como se puede apreciar a continuación: "Artículo 8. La corporación de Guardias de Seguridad Industrial, Bancaria y Comercial y la de Vigilantes Auxiliares dependerán de la Dirección General de Seguridad Pública y Tránsito del Estado, al frente de dichas corporaciones se designará un jefe”.

En algún momento de la historia y con la finalidad de alejar de todo escrutinio los ingresos y operación de este organismo, se tomó una ruta recorrida simultáneamente por dos carriles. El primero, en el que para ofer-

2 "Regularán policía privada de la ASE", disponible en El Universal (Alonso, 2011): http://archivo.eluniversal.com.mx/ciudad/105478.html. 
Esta revista forma parte del acervo de la Biblioteca Jurídica Virtual del Instituto de Investigaciones Jurídicas de la UNAM

tar los servicios y mantener una relación de confianza con los clientes, los elementos de la corporación detentan una condición de instancia gubernamental que se refleja en oficios, contratos y páginas de Internet ${ }^{3}$ en las que constantemente se emplean logotipos y escudos oficiales del gobierno del Estado de México y se hace referencia a la condición de elementos sujetos al apartado B del artículo 123 de la Constitución federal. En el segundo carril, los vacíos legales provocados por las constantes reformas a la legislación local en materia de seguridad pública favorece la pretensión de negar toda relación de dependencia y control, sobre todo cuando de lo que se trata es de requerir las cuentas de su funcionamiento, llegando a formar parte de esta maquinación la constante modificación de la propia denominación de dicho organismo auxiliar. Esto nos recuerda que en no pocas ocasiones los funcionarios pueden encontrar maneras más sofisticadas y difíciles de detectar para evadir las demandas de transparencia (Roberts, 2009: 197).

\section{La RELACión ADMinistrativa QUE EXISTE}

Contrario a lo resuelto por la mayoría, en los votos disidentes que he formulado, he señalado que la seguridad pública es una función a cargo de la Federación, los estados y municipios, según lo establecido en el noveno párrafo del artículo 21 de la Constitución Política de los Estados Unidos Mexicanos, lo que en el Estado de México es una función a cargo del estado y los municipios, según lo dispone el artículo 86 bis de la Constitución local y el 2o. de la ley estatal.

Para definir el concepto de seguridad pública debemos partir del deber del Estado como encargado de vigilar el orden público, garantizar la paz y la seguridad de la comunidad (González, 2002: 126). Dicha función se relaciona con la preservación de determinados valores tutelados, entre los cuales se encuentran los de la vida e integridad de las personas, sus derechos y libertades, el orden y la paz públicos (González, 2002: 128).

La Secretaría de Seguridad Ciudadana, primero, y la Comisión Estatal de Seguridad Ciudadana, después, han señalado reiteradamente que los Cuerpos de Seguridad Auxiliares del Estado de México cuentan con un régimen jurídico que se desprende del artículo 103, segundo párrafo, de la Ley de Seguridad del Estado; por lo tanto, se trata de un organismo a través del cual el Estado proporciona servicios de protección, custodia, vi-

\footnotetext{
3 Como puede ser observado y consultado en http://www.region-6.com.mx/index.htm.
} 
Esta revista forma parte del acervo de la Biblioteca Jurídica Virtual del Instituto de Investigaciones Jurídicas de la UNAM

gilancia y seguridad a sociedades mercantiles, asociaciones, instituciones educativas, particulares, dependencias y organismos públicos. Dichos servicios contribuyen a la preservación del orden y la paz públicos y tienden a asegurar el respeto a la vida, a la integridad y a los derechos y libertades de las personas que cotidianamente acuden a las instalaciones de las dependencias públicas; por lo tanto, las actividades que realizan los Cuerpos de Seguridad mencionados forman parte de la función general de seguridad pública.

De lo anterior podría deducirse que quien brinda los servicios de protección, custodia, vigilancia y seguridad señalados no es el Estado directa- mente. Por ello, en los recursos de revisión 1129 y 1130 del 2015, el Pleno

- del órgano garante confirmó la respuesta bajo el argumento de que sus integrantes no son servidores públicos. Bajo esa tesitura, dicho organismo sería un particular autorizado por el Estado para brindar los mencionados servicios, interpretación que la propia autoridad promueve al señalar que estos organismos están integrados en su totalidad por particulares y no por servidores públicos, y que no reciben recursos del presupuesto público asignado a la Comisión Estatal de Seguridad Ciudadana; bajo ese criterio, podría decirse que este tipo especial de seguridad privada realiza actividades auxiliares en materia de seguridad pública.

Sin embargo, al resolverse el recurso de revisión 723, el proyecto rechazado por la mayoría pretendía acreditar que se trata de un servicio privado de seguridad, por lo que la Comisión Estatal de Seguridad Ciudadana cuenta con facultades suficientes de control que le permitirían contar con información relacionada no sólo con el número de sus integrantes y el nombre del titular, sino sobre el aspecto más importante y que mayor interés provoca en la sociedad: los servicios que ofrece, sus tarifas y, en consecuencia, los ingresos que percibe. Con el rechazo mayoritario al proyecto y con la aprobación de la resolución final, la mayoría confirmó que dicho organismo no forma parte de la administración estatal y, al mismo tiempo, no es un particular en funciones de seguridad, apartándose — sin proporcionar mayores razones - de su anterior criterio.

Pero lo que la mayoría del Pleno del órgano garante del Estado de México no puede desvirtuar es que la función de seguridad pública corresponde a los tres niveles de gobierno, y, en el caso del Estado de México, los servicios de protección, custodia, vigilancia y seguridad a dependencias y organismos públicos, sociedades mercantiles y demás sujetos referidos previamente, pueden ser brindados por el estado a través de organismos que tienen el carácter de auxiliares de la función de seguridad pública y 
Esta revista forma parte del acervo de la Biblioteca Jurídica Virtual del Instituto de Investigaciones Jurídicas de la UNAM

que se integran por personal que, aunque a dicho de la Comisión Estatal de Seguridad Ciudadana, no cuenta con la condición de servidores públicos, en los diversos contratos firmados por el organismo auxiliar y las autoridades federales (la Comisión Nacional para la Protección y Defensa de los Usuarios de Servicios Financieros y el Banco Nacional de Obras y Servicios Públicos, por citar un par de ejemplos), dicha corporación ha declarado que sus integrantes se rigen por el apartado B del artículo 123 de la Constitución Política de los Estados Unidos Mexicanos, que sólo es aplicable precisamente a los trabajadores al servicio del Estado.

Cuando el sujeto obligado se refiere a lo dispuesto en el segundo párrafo del artículo 103 de la Ley de Seguridad del Estado de México, en la fracción XV del artículo 8o. de la Ley que crea la Comisión Estatal de Seguridad Ciudadana del Estado de México, y en la fracción XXII del artículo 10 del Reglamento Interior de la Comisión Estatal de Seguridad Ciudadana para deducir de ello que la relación que existe es estrictamente operativa y en situaciones de urgencia, realiza una lectura parcial, limitada y defectuosa de los ordenamientos jurídicos que, como dependencia especializada, debería de operar con mayor claridad y consistencia. Lo anterior en virtud de que es función del secretario de seguridad ciudadana coordinar tanto a las instituciones policiales estatales como a los organismos en cuestión, según lo señala el artículo 16, fracción IV, de la Ley de Seguridad del Estado de México, y aun las propias disposiciones citadas por el sujeto obligado, entre ellas el segundo párrafo del artículo 103 de esta misma ley, la cual refiere que la "organización y funcionamiento de estos cuerpos de seguridad, se regularán por las disposiciones administrativas que emitan las dependencias del Gobierno del Estado". Entre esas disposiciones administrativas se encuentra el Reglamento Interior de la Comisión Estatal de Seguridad Ciudadana, al que también se refirió el sujeto obligado, señalando que su relación se limita a coordinar operativamente a dichos organismos auxiliares, según la fracción XXII del artículo 10 del Reglamento en cuestión, pero omitió señalar que las dos fracciones previas de la porción legal aludida por el sujeto obligado facultan a la misma dependencia para:

XX. Regular, coordinar, supervisar y controlar a los prestadores de servicios de seguridad privada y verificar el cumplimiento de las disposiciones normativas en la materia, así como recibir, atender y resolver las quejas sobre su actuación.

XXI. Realizar el registro, seguimiento y control del personal de la Comisión y de los prestadores del servicio de seguridad privada, así como el de los cuerpos preventivos de seguridad pública municipal. 
Esta revista forma parte del acervo de la Biblioteca Jurídica Virtual del Instituto de Investigaciones Jurídicas de la UNAM

No está por demás señalar que la fracción primera de dicho artículo faculta a la Comisión para "coordinar los servicios de seguridad pública y de prevención del delito al interior del estado y salvaguardar la integridad, los derechos y bienes de las personas, así como preservar la libertad, el orden y la paz públicos". De la lectura de estas disposiciones puede apreciarse que la Comisión Estatal coordina a todos los cuerpos de seguridad pública; registra, da seguimiento y controla al personal tanto de dicha dependencia como de los cuerpos preventivos de seguridad pública municipal, todos los anteriores integrados por servidores públicos; asimismo, regula, coordina, supervisa y controla a los organismos integrados por particulares que no son servidores públicos y que, en consecuencia, brindan servicios de seguridad privada. Por lo tanto, si realiza dichas acciones en el caso de los servidores públicos en funciones policiales, en uno de los extremos del caso, y también de los particulares que brindan el servicio de seguridad, en el otro extremo, es evidente, y la sana lógica nos dicta, que la relación que existe entre la Comisión Estatal con los denominados Cuerpos de Seguridad Auxiliares del Estado de México se encuentra perfectamente comprendida dentro de sus facultades de control y supervisión.

Por otro lado, la Ley de Seguridad del Estado de México determina procedimientos claros, precisos y permanentes para favorecer el desempeño personal en materia de desarrollo policial, lo que según el artículo 134 de la ley, cuya iniciativa fue presentada por el gobernador del estado, tenía "por objeto garantizar el desarrollo institucional, la estabilidad, la seguridad y la igualdad de oportunidades de los mismos; elevar la profesionalización, fomentar la vocación de servicio y el sentido de pertenencia, así como garantizar el cumplimiento de los principios" a que se refiere la ley. Estos fines superiores serían inútiles e irrealizables si la autoridad hubiera declinado ejercer sus competencias para controlar y supervisar a quienes integran a estos organismos auxiliares; en consecuencia de ello, y contrario a lo señalado por el sujeto obligado y respaldado por la mayoría del Pleno del Infoem, mantener el seguimiento del funcionamiento de estos organismos se encuentra perfectamente encuadrado en las facultades que el artículo 16, fracción VI, de la multicitada Ley de Seguridad le otorgan al secretario, hoy comisionado de seguridad ciudadana.

Con esas respuestas, el Estado demuestra que ha abdicado al ejercicio de funciones de control y supervisión, dejando en total estado de independencia a un organismo auxiliar con el que comparte la licencia para la portación de armas de fuego, al que autorizó para ejercer dicha función y el que constantemente se detenta como parte de las instituciones del Estado. Esto ha sido suficiente para que la mayoría del órgano garante respalde 
Esta revista forma parte del acervo de la Biblioteca Jurídica Virtual del Instituto de Investigaciones Jurídicas de la UNAM

las respuestas del sujeto obligado, en el sentido de que no existe ninguna relación que no sea la operativa y en casos de desastres, a pesar de que el estudio de las disposiciones jurídicas aplicables a cada caso demuestran que esto no es así.

Con decisiones como éstas, el derecho de acceso a la información pública, lejos de convertirse en un instrumento eficaz para acceder a la verdad, sucumbe ante lo que la Suprema Corte de Justicia de la Nación consideró como intentos para lograr la impunidad de las autoridades, razonamiento consignado en la emblemática investigación del caso Aguas Blancas, y de la que se desprende el siguiente criterio:

Garantías individuales (derecho a la información). Violación grave PREVISTA EN EL SEGUNDO PÁRRAFO DEL ARTICULO 97 CONSTITUCIONAL. LA CONFIGURA EL INTENTO DE LOGRAR LA IMPUNIDAD DE LAS AUTORIDADES QUE ACTÚAN DENTRO DE UNA CULTURA DEL ENGAÑO, DE LA MAQUINACIÓN Y DEL OCULTAMIENTO, POR INFRINGIR EL ARTICULO 6O. TAMBIÉN CONSTITUCIONAL. El artículo 6o. constitucional, in fine, establece que "el derecho a la información será garantizado por el Estado”. Del análisis de los diversos elementos que concurrieron en su creación se deduce que esa garantía se encuentra estrechamente vinculada con el respeto de la verdad. Tal derecho es, por tanto, básico para el mejoramiento de una conciencia ciudadana que contribuirá a que ésta sea más enterada, lo cual es esencial para el progreso de nuestra sociedad. Si las autoridades públicas, elegidas o designadas para servir y defender a la sociedad, asumen ante ésta actitudes que permitan atribuirles conductas faltas de ética, al entregar a la comunidad una información manipulada, incompleta, condicionada a intereses de grupos o personas, que le vede la posibilidad de conocer la verdad para poder participar libremente en la formación de la voluntad general, incurren en violación grave a las garantías individuales en términos del artículo 97 constitucional, segundo párrafo, pues su proceder conlleva a considerar que existe en ellas la propensión de incorporar a nuestra vida política, lo que podríamos llamar la cultura del engaño, de la maquinación y de la ocultación, en lugar de enfrentar la verdad y tomar acciones rápidas y eficaces para llegar a ésta y hacerla del conocimiento de los gobernados (Tesis P. LXXXIX/96).

\section{LA AFECTACión GENERADA AL DERECHO}

A LA PROTECCIÓN DE DATOS PERSONALES

Pero más allá de que lo anterior significa una grave afectación al sistema de rendición de cuentas, la resolución del recurso de revisión 2014, antes referida, adquiere una relevancia significativa. No se trata del debate sobre 
Esta revista forma parte del acervo de la Biblioteca Jurídica Virtual del Instituto de Investigaciones Jurídicas de la UNAM

los recursos, sobre el desempeño de la función pública, sobre el uso o no de armas, sobre la logística y las funciones que esta corporación desempeña. Despejemos por un momento todos estos aspectos y limitemos el análisis sólo al caso en cuestión y apreciaremos que se trata de una persona que desde la obscuridad y la adversidad de la privación de la libertad en la que se encuentra, de manera respetuosa, deferente, comedida, pretende ejercer sus derechos tratando de acceder a sus personalísimos datos para hacer uso de ellos como mejor le parezca.

El artículo 16 de la Constitución Política de los Estados Unidos Mexicanos establece que "[t]oda persona tiene derecho a la protección de sus datos personales, al acceso... en los términos que fije la ley". En el régimen constitucional de derecho que prevalece en las sociedades occidentales, de las que México forma parte, un derecho reconocido constitucionalmente debe ser garantizado independientemente de que exista o no el adecuado desarrollo legislativo, ya que la dignidad de la persona no puede verse afectada por la deliberada omisión del legislador ordinario que pretenda, ante la falta de desarrollo legislativo, negar la efectividad del mismo (Sagüés, 2013: 192). En el caso del derecho a la protección de datos personales, la situación jurídica no es homogénea ni la más adecuada, pero tampoco se encuentra en un estado completo de indefensión que impida su racional y adecuada protección. Como he señalado, el marco constitucional ha reconocido el derecho a la protección de los datos personales y las diferentes modalidades de su ejercicio, entre ellas, el acceso a los mismos, remitiendo a la legislación secundaria la forma en la que ha de ejercerse.

Partamos de una verdad evidente: los datos personales pueden encontrarse en posesión de las autoridades o de los particulares. Para garantizar su adecuada protección estamos en vísperas de que el Congreso de la Unión emita la Ley General de Datos Personales en Posesión de Sujetos Obligados, ordenada en la reforma constitucional de 2014. Pero aun cuando en este momento carecemos de dicha ley general, existen medios legales mínimos que permiten una adecuada tutela que, desde luego, se verá fortalecida con la ley general en su momento.

De ser el caso que los datos personales de un mexiquense se encuentren en posesión de las autoridades, puede aplicarse el artículo 16 de la Ley Federal de Transparencia, lo que no se aplicaba en dicho recurso de revisión, toda vez que se trata de un cuerpo auxiliar de carácter estatal con el que la autoridad de nuestra entidad tiene relación, aunque sólo sea, suponiendo sin conceder, en materia de desastres.

Si los datos personales se encontraran en posesión de la autoridad estatal, aplicaría perfectamente la Ley de Protección de Datos Personales 
Esta revista forma parte del acervo de la Biblioteca Jurídica Virtual del Instituto de Investigaciones Jurídicas de la UNAM

del Estado de México, vigente desde el 1o. de septiembre de 2012. Pero quienes integraron la mayoría que aprobó la resolución nos dicen que no se encuentra en posesión de una autoridad estatal.

Si dichos datos no se encuentran en posesión de autoridades federales o estatales, la única posibilidad que existe es que se encuentren en posesión de un particular y sería razonablemente lógico que la resolución señalara que el ordenamiento jurídico aplicable era la Ley Federal de Protección de Datos Personales en Posesión de Particulares, y la autoridad facultada para su tutela, el Instituto Nacional de Acceso a la Información y Protección de Datos Personales. Si la mayoría hubiera sido consecuente con otros criterios que ha sostenido antes en esta materia, hubiera sido perfectamente lógico y razonable, incluso casi aceptable, que hubiera señalado lo anterior.

Pero la mayoría se apartó de sus criterios pasados y no quiso asumir dicha conclusión, porque ello implicaría que dado que el marco normativo de la Comisión Estatal de Seguridad Ciudadana le alcanza para controlar a los particulares que brindan servicios de seguridad, en otras solicitudes de acceso a la información estaría obligada a entregar información que se ha negado sistemáticamente a entregar.

\section{Consecuencias de las Resoluciones del óRgano garante}

Las resoluciones emitidas son a todas luces contrarias a la tendencia general que se experimenta en el país por transparentar la función pública y sujetar a control los actos de gobierno, lo que permite impedir actos de corrupción que afectan la prestación de bienes y servicios públicos (Cossío, 2008: 113). Las resoluciones lesionan gravemente el Estado constitucional de derecho en la materia porque establecen un marco de opacidad inexpugnable en casos que involucran cuantiosos recursos económicos, sobre el desempeño y funcionamiento de una corporación vinculada con temas de seguridad pública y cuyos elementos se han visto involucrados en hechos delictivos; de esta forma, un organismo auxiliar del Estado, cuya creación y existencia se encuentra claramente establecida en la ley, se coloca en una condición de cuerpo paramilitar ajeno a cualquier control de las autoridades democráticas. Afecta gravemente el Estado constitucional de derecho, ya que al negarse a proteger los derechos de acceso a los datos personales, ubica a dichos Cuerpos Auxiliares en un espacio ajeno al control de las autoridades. Tanto la primera como la segunda consecuencia debilitan el esfuerzo 
Esta revista forma parte del acervo de la Biblioteca Jurídica Virtual del Instituto de Investigaciones Jurídicas de la UNAM

generalizado por combatir la corrupción y el uso discrecional de los recursos públicos.

\section{Fuentes de información}

Alfa Diario, 2016, "Insisten en ocultar información de Cusaem", febrero, disponible en: http:/wwww.alfadiario.mx/articulo/2016-02-28/63428/ gobierno-insiste-en-ocultar-informacion-de-cusaem.

Alonso, Eduardo, 2011, "Regularán policía privada de la ASE", El Universal, 13 de marzo, disponible en: http://archivo.eluniversal.com.mx/ciudad/ 105478.html.

Cossío Díaz, José Ramón, 2008, “Transparencia y Estado de derecho”, en Ackerman, John M. (coord.), Más allá del acceso a la información. Transparencia, rendición de cuentas y estado de derecho, México, Siglo XXI.

El INFORMADOR, 2011, "Sedena investigará destino de armamento entregado a ASE y Cusaem", marzo, disponible en: http://www.informador.com. mx/mexico/2011/277399/6/sedena-investigara-destino-de-armamento-en tregado-a-ase-y-cusaem.htm.

GonzÁlez Fernández, José Antonio, 2002, "La seguridad pública en México”, en Peñaloza, Pedro José y Garza Salinas, Mario A. (coords.), Los desafíos de la seguridad pública en México, México, Procuraduría General de la República-UNAM, Instituto de Investigaciones Jurídicas, disponible en: http://biblio.juridicas.unam.mx/libros/1/419/12.pdf.

Murillo de la Cueva, Pablo Lucas, 1993, Informática y protección de datos personales, Madrid, Centro de Estudios Constitucionales.

Poder Legislativo del Estado de México, 2015, Gaceta Parlamentaria, 26 de noviembre, disponible en: $h t t p: / / w w w . s e c r e t a r i a d e a s u n t o s p a r l a m e n t a r i o s$. gob.mx/mainstream/Actividad/Gaceta/LIX/GP-13\%20(26-NOV-15).pdf .

Roberts, Alaisdar, 2009, "La lucha por gobiernos abiertos", en Sandoval, Irma Eréndira (coord.), Corrupción y transparencia. Debatiendo las fronteras entre Estado, mercado y sociedad, México, Siglo XXI-UNAM, Instituto de Investigaciones Sociales.

SagüÉs, Néstor Pedro, 2013, La interpretación judicial de la Constitución. De la Constitución nacional a la Constitución convencionalizada, México, Porrúa-Instituto Mexicano de Derecho Procesal Constitucional.

Tesis P. LXXXIX/96, Semanario Judicial de la Federación y su Gaceta, Pleno, Novena Época, t. III, junio de 1996. 
Esta revista forma parte del acervo de la Biblioteca Jurídica Virtual del Instituto de Investigaciones Jurídicas de la UNAM http://www.juridicas.unam.mx/

DOI: http://dx.doi.org/10.22201/iij.25940082e.2017.4.11521

https://revistas.juridicas.unam.mx/

LOS CUERPOS AUXILIARES DE SEGURIDAD PÚBLICA DEL ESTADO DE MÉXICO...

Villamil, Jenaro, 2013, "Cusaem, la corporación policiaca al servicio de los políticos", junio, disponible en: https://jenarovillamil.wordpress. com/2013/06/16/cusaem-la-corporacion-policiaca-al-servicio-de-los-politi cos-primera-partel. 